\title{
Essential Oil from Bush Mint, Hyptis suaveolens, is as Effective as DEET for Personal Protection against Mosquito Bites
}

\author{
A.Z. Abagli and T.B.C. Alavo* \\ Laboratoire d'Entomologie appliquée, Faculté des Sciences et Techniques (FAST), Université d'Abomey-Calavi (UAC), \\ Bénin
}

\begin{abstract}
Concern about the deleterious effects associated with synthetic chemicals has revived interest to explore plants as a source of natural insecticides for mosquito control. Ethnobotanical studies conducted in Kenya on plant species including bush mint, Hyptis suaveolens Poit., showed that many of them repel mosquitoes effectively when burned overnight in rooms. Recent field works conducted with $H$. suaveolens essential oil have demonstrated the potential of this essential oil as mosquito repellent. The present work is a comparative study on the persistence of $30 \%$ DEET and $10 \% H$. suaveolens essential oil for personal protection against mosquitoes in field conditions. Twenty volunteers who have given their informed consent have been involved for each of the products and control (no treatment). Results showed that the mean number of mosquitoes that landed on treated volunteers 6 hours post-application was 0.50 and 0.45 for $10 \% H$. suaveolens essential oil and DEET respectively, against 6 mosquitoes for the control people. Statistical analysis revealed that there is no significant difference between $10 \% \mathrm{H}$. suaveolens essential oil and DEET indicating that both products are similarly effective. The possibility to use H. suaveolens essential oil as integrated malaria vector management has been discussed.
\end{abstract}

Keywords: DEET, Hyptis suaveolens, essential oil, repellent, mosquito control.

\section{INTRODUCTION}

Chemical repellents are important in protecting people from blood-feeding insects, ticks, mites, and other arthropods and may therefore also reduce transmission of arthropod-borne diseases [1]. $N, N$-diethyl-3-methylbenzamide (DEET) is one of the most well-known arthropod repellents and has been on the market for almost half a century $[2,3]$. DEET is effective against many different blood-sucking arthropods $[2,4]$. The protection efficacy depends on the type of formulation, application pattern, species, and feeding behavior of the arthropod [4]. DEET is generally safe for topical use if applied as recommended, although adverse effects such as serious neurologic effects have been reported $[4,5]$. Many people consider that DEET and related compounds are a health and environmental hazard [6]. DEET does not readily degrade by hydrolysis at environmental $\mathrm{pHs}$ and has been identified as a ubiquitous pollutant in aquatic ecosystems [6,7]. Concern about the deleterious effects associated with synthetic chemicals has revived interest to explore plants as a source of natural insecticides, acaricides, and repellents for medical, veterinary and crop protection use [1].

Ethnobotanical studies conducted in Kenya on plant species including Hyptis suaveolens Poit. showed that many of them repel mosquitoes effectively when burned overnight in rooms [8]. Duke [9] also includes $H$. suaveolens in his

*Address correspondence to this author at the Laboratoire d'Entomologie appliquée, Faculté des Sciences et Techniques (FAST), Université d'Abomey-Calavi (UAC), BP 215 Godomey, Bénin; Tel: (229) 97875438; E-mail: thieryalavo@hotmail.com phytochemical and ethnobotanical database as an insect repellent. Laboratory study has assessed the repellency rates of various concentrations of $H$. suaveolens essential oil and $6 \%$ of the oil was said to induce a high repellency rate in laboratory conditions [10]. Recent field works conducted with $H$. suaveolens essential oil showed that the effects of a solution containing $8 \%$ of the oil persisted and repelled up to $97.56 \%$ of mosquitoes by 5 hours post-application [11]. Here we report results of a comparative study carried out in field conditions on $10 \% \mathrm{H}$. suaveolens essential oil and $\mathrm{N}, \mathrm{N}$ diethyl-3-methylbenzamide (DEET).

\section{MATERIALS AND METHODS}

\section{Extraction of $\boldsymbol{H}$. suaveolens Essential Oil}

The extraction of $H$. suaveolens essential oil was made from leaves collected from plants cultivated according to Ahoton et al. [12]. The harvested leaves were air-dried in the shade for three days. The extraction of the essential oil was made by steam distillation using $1 \mathrm{~m}^{3}$ still. To carry out the tests, the essential oil was dissolved in isopropanol $(99.8 \%$ pure).

\section{Study Areas}

The field works took place in Ladji, Towéta and Vossa districts located in Cotonou (Benin, West Africa). These are wetlands and floodable locations without modern infrastructures and contain many mosquitoes breeding sites. These locations are unhealthy and unfit for human accommodation, nevertheless thousands peoples live there in poor health and social conditions. 
Field Study on the Effect of $10 \%$ H. suaveolens Essential Oil Immediately after Application

To study the effect of $10 \% H$. suaveolens essential oil immediately post-application, a total of 20 replicates were carried out through the study areas. The product was applied directly on both feet (from toes to knee) of each of the volunteers who gave their informed consent. The application was made using cotton soaked in the essential oil solution. For the observations, treated volunteer and the control (untreated person) were installed at a distance of about $3 \mathrm{~m}$ from each other on a stool. Mosquitoes coming to rest on the feet of the volunteers were then captured using a mouth vacuum during a period of 15 minutes. The collected mosquitoes are brought to the laboratory for counting and identification, using a stereomicroscope (Motic China). The experiments have been carried out between $8 \mathrm{pm}$ and $10 \mathrm{pm}$ in May-June 2010.

\section{Comparative Study on the Persistence of $10 \% H$.} suaveolens Essential Oil and DEET in Field Conditions

The persistence of the effect of $10 \% H$. suaveolens essential oil as well as DEET has been investigated 6 hours post-application. To assess the persistence of these products on mosquito populations after this period of time, the feet of volunteers were treated 6 hours before the start time of the observations in the study areas. Twenty replicates were performed for each product and the control (untreated volunteers). The field observations and mosquito counts were made in the same manner as described above. These experiments have been carried out in July-August 2010. The commercial formulation of N, N-diethyl-3-methylbenzamide (DEET) called 'Ungava' has been used. 'Ungava' contains $30 \%$ DEET and is manufactured by the Company 'Aerokure International Inc.' (Canada).

\section{Statistical Analyses}

Non-parametric tests (Mann-Whitney U) were performed to determine whether there is significant difference between the number of mosquitoes coming to rest on the feet of volunteers in tested variants. These tests were performed since the data did not meet the ANOVA hypotheses. Statistical analyses have been performed using SPSS statistics package version 16.0.

\section{RESULTS}

\section{Effect of $10 \%$ H. suaveolens Essential Oil Immediately} after Application

The total number of mosquitoes that landed on treated and control feet during the first 15 minutes post-application in all replicates is 0 and 375, respectively (Fig. 1). In terms of percentage, these results show that $100 \%$ of the mosquitoes were repelled the first 15 minutes post-application of the solution containing $10 \%$ of $H$. suaveolens essential oil. Statistical analyses showed that there is significant difference between essential oil treated volunteers and the control. Two species of feeding female mosquitoes were captured and identified namely Culex quinquefasciatus and Anopheles gambiae; the Culex mosquitoes prevailing, however, in the study areas (Table 1).

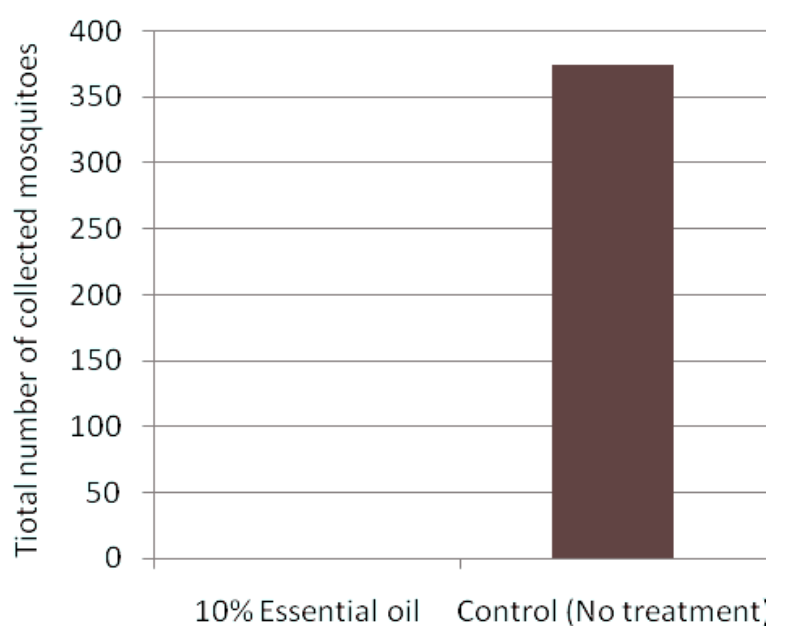

Fig. (1). Effect of $10 \% H$. suaveolens essential oil on mosquitoes populations in field conditions immediately post-application.

Table 1. Biodiversity and Number of Collected Mosquitoes on Untreated Controls Immediately after Application of $10 \%$ H. suaveolens Essential Oil

\begin{tabular}{|c|c|}
\hline Mosquito species & Number of collected individuals \\
\hline \hline Culex quinquefasciatus & 367 \\
\hline Anopheles gambiae & 8 \\
\hline Aedes $\mathrm{sp}$. & 0 \\
\hline
\end{tabular}

\section{Persistence of the Effect of $10 \%$ Essential Oil and DEET in Field Conditions}

The mean number of mosquitoes that landed on treated volunteers 6 hours post-application was 0.50 and 0.45 for $10 \%$ H. suaveolens essential oil and DEET respectively, against 6 mosquitoes for the control (Fig. 2). This corres-

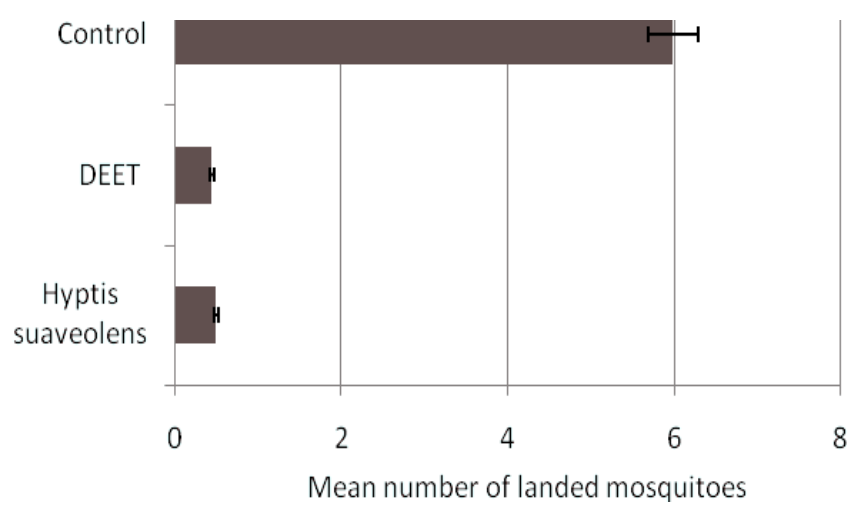

Fig. (2). Mean number of collected mosquitoes on treated volunteers 6 hours post-application.

ponds to a repellency rate of about $92 \%$ for both products. Statistical analyses showed that there is no significant difference between $10 \% H$. suaveolens essential oil and DEET indicating that both products are similarly effective. 
During these field experiments, Culex, Anopheles and Aedes mosquitoes have been captured on treated and untreated volunteer feet; Culex mosquitoes prevailing, however, in the study areas (Table 2).

Table 2. Biodiversity and Number of Mosquitoes Collected on Treated and Control Volunteers in all Field Trials 6 Hours Post-Application

\begin{tabular}{|c|c|c|c|}
\hline Treatment & Culex sp. & Anopheles sp. & Aedes sp. \\
\hline \hline Hyptis & 10 & 0 & 0 \\
\hline DEET & 8 & 0 & 1 \\
\hline Control & 102 & 8 & 4 \\
\hline
\end{tabular}

\section{DISCUSSIONS}

The concentration of $6 \%$ of $H$. suaveolens essential oil produced the best results in laboratory tests since it has repelled about $97 \%$ of tested mosquitoes. When tested in field conditions, this concentration repelled $100 \%$ of mosquitoes present in the test areas the first 15 minutes postapplication [11]. In the present study, $10 \% \mathrm{H}$. suaveolens essential oil induced also the maximal repellency rate. This confirms once again that low concentration of this essential oil is highly effective against mosquito populations, the first hour post-application. Comparative study conducted on the efficacy of insect repellents against mosquito bites demonstrated that higher concentrations of DEET provided longerlasting protection [13]. As for DEET, higher concentration of $H$. suaveolens essential oil provided also longer-lasting protection [11]. In the present study, $10 \% \mathrm{H}$. suaveolens essential oil and a formulation containing 30\% DEET provided similar protection time amounting to at least 5 hours. Our data are in agreement with Fradin and Day [13] results that revealed a mean complete protection time of 5 hours with a formulation containing 23.8\% DEET. Based on these data, we conclude that $10 \% H$. suaveolens essential oil is as effective as $30 \%$ DEET for personal protection against mosquito bites.

The majority of mosquitoes captured in the present study on untreated volunteers were predominantly Culex quinquefasciatus and occasionally Anopheles gambiae, the major malaria vector in Sub-Saharan Africa. Laboratory works have also demonstrated that low concentration (6\%) of $H$. suaveolens essential oil induced maximal repellency rate against $A$. gambiae [10]. In Sub-Saharan Africa, it is actively recommended to people to spend night in impregnated mosquito nets in order to avoid malaria infection. Nevertheless, people who usually sleep under insecticidal nets, still contract malaria from time to time. For instance, in a study conducted in Somalia, it was demonstrated that the protective efficacy of insecticidal nets against malaria transmission is barely $54 \%$ among people who regularly use mosquito nets [14]. Moreover, Toe-Pare et al. [15] have shown that people's motivation to use mosquito nets considerably decreased less than a year after the campaigns and people prefer to spend night without insecticidal nets. Therefore, mosquito net is not sufficient to effectively control malaria vectors, especially in the West African countries where urbanization promotes the proliferation of mosquitoes [16]. Consequently, to achieve successful vectors control and reduce substantially the prevalence of malaria and other vector-borne diseases, an integrated management of these vectors must be adopted as recommended Okech et al. [17]. In this perspective, the present study shows that a formulation containing $10 \%$ essential oil of $H$. suaveolens is a way that may be taken into account for the integrated management of disease-vectors mosquitoes.

\section{AKNOWLEGEMENTS}

This work has been supported by the Rectorate of the University of Abomey-Calavi, Benin. The participation of unpaid volunteers in the present study is highly appreciated.

\section{REFERENCES}

[1] Jaenson TGT, Palsson K, Borg-Karlson AK. Evaluation of extracts and oils of mosquito (Diptera: Culicidae) repellent plants from Sweden and Guinea-Bissau. J Med Entomol 2006; 43(1): 113-19.

[2] Brown M, Hebert AA. Insect repellents: an overview. J Am Acad Dermatol 1997; 36: 243-49.

[3] Fradin MS. Mosquitoes and mosquito repellents: a clinician's guide. Ann Intern Med 1998; 128: 931-40.

[4] Qiu H, Jun HW, McCall JW. Pharmacokinetics, formulation, and safety of insect repellent $N, N$-dietyl-3-methylbenzamide (DEET): a review. J Am Mosq Control Assoc 1998; 14:12-27.

[5] Sudakin DL, Trevathan WR. DEET: a review and update of safety and risk in the general population. J Toxicol Clin Toxicol 2003; 41: 831-9.

[6] Aquino M, Fyfe M, MacDougall L, Remple V. West Nile virus in British Columbia. Emerg Infect Dis 2004; 10: 1499-501.

[7] Weigel S, Kuhlmann J, Huhnerfuss H. Drugs and personal care products as ubiquitous pollutants: occurrence and distribution of clofibric acid, caffeine and DEET in the North Sea. Sci Total Environ 2002; 295: 131-41.

[8] Seyoum A, Kabiru EW, Lwande W, Killeen GF, Hassanali A, Knols BG. Repellency of live potted plants against Anopheles gambiae from human baits in semi-field experimental huts. Am J Trop Med Hyg 2002; 67 (2): 191-5.

[9] Duke JA. Dr. Duke's phytochemical and ethnobotanical databases. (http://www.ars-grin.gov/cgi-bin/duke/ethnobot.pl). Consulted 2007 on $11 / 04 / 2007$

[10] Abagli AZ, Alavo TBC, Djouaka R, et al. Taux de répulsion de différentes concentrations de l'huile essentielle de Hyptis suaveolens contre le moustique Anopheles gambiae (Diptera: Culicidae). Actes du $2^{\mathrm{e}}$ colloque de l'UAC des Sciences, Cultures et Technologies, Sciences agronomiques 2009; pp. 329-49.

[11] Abagli AZ, Alavo TBC, Avlessi F, Moudachirou M. Potential of Hyptis suaveolens essential oil for personal protection against mosquitoes (Diptera : Culicidae). Personal Communication 2011.

[12] Ahoton LE, Alavo TBC, Ahomadegbe MA, Ahanhanzo C, Agbangla C. Domestication du gros baume (Hyptis suaveolens (L.) Poit.): Techniques de production et potentiels insectes ravageurs au sud du Bénin. Int J Biol Chem Sci 2010; 4 (3): 608-14.

[13] Fradin MS, Day FJ. Comparative efficacy of insect repellents against mosquito bites. N Engl J Med 2002; 347 (1): 13-8.

[14] Noor AM, Moloney G, Borle M, Fegan GW, Shewchuk T, Snow $\mathrm{RW}$. The use of mosquito nets and the prevalence of plasmodium infection in rural South Central Somalia. PLoS One 2008; 3(5): e2081.

[15] Toe-Pare L, Skovmand O, Damir KR, et al. Decreased motivation in the use of insecticide-treated nets in malaria endemic area in Burkina Faso. Malar J 2009; 8(1): 175. 
[16] Alavo TBC, Abagli AZ, Accodji M, Djouaka R. Unplanned urbanization promotes the proliferation of disease vector mosquitoes (Diptera: Culicidae). Open Entomol J 2010; 4: 1-7.
[17] Okech BA, Mwobobia IK, Kamau A, et al. Use of integrated malaria management reduces malaria in Kenya. PLoS One 2008; 3(12): e4050.

Received: March 14, 2011

(C) Abagli and Alavo; Licensee Bentham Open.

This is an open access article licensed under the terms of the Creative Commons Attribution Non-Commercial License (http://creativecommons.org/licenses/by$\mathrm{nc} / 3.0 /$ ), which permits unrestricted, non-commercial use, distribution and reproduction in any medium, provided the work is properly cited. 